\title{
Burst-like control of lipolysis by the sympathetic nervous system in vivo
}

\author{
Katrin Hücking, Marianthe Hamilton-Wessler, Martin Ellmerer, and Richard N. Bergman \\ Department of Physiology and Biophysics, Keck School of Medicine, University of Southern California, Los Angeles, \\ California, USA
}

\begin{abstract}
Rapid oscillations of visceral lipolysis have been reported. To examine the putative role of the CNS in oscillatory lipolysis, we tested the effects of $\beta_{3}$-blockade on pulsatile release of FFAs. Arterial blood samples were drawn at 1 -minute intervals for 120 minutes from fasted, conscious dogs $(n=7)$ during the infusion of saline or bupranolol $(1.5 \mu \mathrm{g} / \mathrm{kg} / \mathrm{min})$, a high-affinity $\beta_{3}$-blocker. FFA and glycerol time series were analyzed and deconvolution analysis was applied to estimate the rate of FFA release. During saline infusion FFAs and glycerol oscillated in phase at about eight pulses/hour. Deconvolution analysis showed bursts of lipolysis (nine pulses/hour) with time-dependent variation in burst frequency. Bupranolol completely removed rapid FFA and glycerol oscillations. Despite removal of lipolytic bursts, plasma FFAs $(0.31 \mathrm{mM})$ and glycerol $(0.06 \mathrm{mM})$ were not totally suppressed and deconvolution analysis revealed persistent non-oscillatory lipolysis $(0.064 \mathrm{mM} / \mathrm{min})$. These results show that lipolysis in the fasting state consists of an oscillatory component, which appears to be entirely dependent upon sympathetic innervation of the adipose tissue, and a non-oscillatory, constitutive component, which persists despite $\beta_{3}$-blockade. The extinction of lipid fuel bursts by $\beta_{3}$-blockade implies a role for the CNS in the maintenance of cyclic provision of lipid fuels.
\end{abstract}

J. Clin. Invest. 111:257-264 (2003). doi:10.1172/JCI200314466.

\section{Introduction}

FFAs are the major oxidative fuel for skeletal muscle, myocardium, liver, and kidney in the fasting state $(1,2)$. Stored in the form of triglycerides in adipose tissue (with less than $5 \%$ in other tissues), FFAs are mobilized via lipolysis (3) when energy demand cannot be met by circulating fuels. The capability of storing and mobilizing energy was fundamental for survival during evolution when a regular intake of food was not guaranteed (4). Today, especially in the industrial world, major health problems are more related to a virtually unlimited excess availability of food, and perturbation of FFA release has been linked to obesity-related diseases including diabetes mellitus (5) and coronary heart disease (6). Since appropriate regulation of FFA availability is critical for metabolic integrity (7), lipolysis requires dynamic regulation.

We have recently demonstrated the existence of powerful and rapid oscillations of lipolysis that are reflected in oscillatory patterns of plasma FFAs and glycerol (8). That FFA oscillations exist independent of insulin cyclicity was supported by evidence that

Received for publication October 22, 2001, and accepted in revised form November 12, 2002.

Address correspondence to: Richard N. Bergman, Department of Physiology and Biophysics, Keck School of Medicine,

University of Southern California, 1333 San Pablo Street, MMR 626, Los Angeles, California 90033, USA. Phone: (323) 442-1920; Fax: (323) 442-1918; E-mail: rbergman@usc.edu.

Conflict of interest: The authors have declared that no conflict of interest exists.

Nonstandard abbreviations used: coefficient of variation (CV); lipoprotein lipase (LPL).
FFA oscillations were not in synchrony with insulin, and using the clamp technique to remove insulin pulses failed to remove or even dampen FFA oscillations (8). Thus it was of interest to investigate other potential sources of oscillations of FFAs in blood.

The recent discoveries of the central melanocortin pathway and centrally acting peptides such as leptin highlight the important role of the CNS in the regulation of food intake and energy expenditure $(9,10)$. Moreover, there is evidence that the CNS may be directly involved in the regulation of fat storage and lipolysis (11). The CNS innervates adipose tissue through efferent pathways of the sympathetic nervous system (12). Sympathetic nervous system innervation of white adipose tissue originates from several regions within the brain stem. These may include the medulla, the nucleus of the solitary tract, or the caudal raphe nucleus; innervation may also originate in the central gray areas located in the forebrain $(13,14)$. Electrical or chemical stimulation of those brain sites leads to increased lipid mobilization, resulting in an elevation of plasma FFA concentration $(15,16)$. However, demonstration that excited regions can induce a generalized response such as lipid mobilization does not clarify which regions are involved in a specific physiologic state. Whether efferent pathways of the CNS play any role in moment-tomoment regulation of fuel provision via breakdown of stored triglycerides remains to be clarified.

The aim of the present study was to test the hypothesis that oscillations of lipolysis are directly controlled by the CNS via its efferent limb to the adipose tissue: the sympathetic nervous system. If this hypothesis were correct, blockade of the sympathetic innervation 
of the adipose tissue would remove cyclic oscillations of lipolysis. To block sympathetic input to the fat cells without affecting additional sympathetic functions, we exploited the high affinity of the $\beta$-blocker bupranolol for the $\beta_{3}$-receptor, which is expressed predominantly in adipose tissue (17). To quantify combined pulsatile and basal release of FFAs in vivo, we used the deconvolution method, which we validated for the assessment of oscillatory FFA release in the canine model used for our experiments.

\section{Methods}

Animals. Experiments were performed on male mongrel dogs housed under controlled kennel conditions at the Keck School of Medicine Vivarium. Animals were used for experiments if judged to be in good health as determined by visual observation, weight stability, body temperature, and hematocrit. The University of Southern California Institutional Animal Care and Use Committee approved all surgical and experimental procedures. The dogs were fed a standard canned food consisting of $27 \%$ protein, $8 \%$ fat, $44 \%$ carbohydrate, and $8 \%$ fiber (Canine $\mathrm{p} / \mathrm{d}$; Hill's Pet Nutrition Inc., Topeka, Kansas, USA) and had free access to a standard dry chow consisting of $26 \%$ protein, $15 \%$ fat, $40 \%$ carbohydrate, and 3\% fiber (Wayne Premium Pet Food; Royal Canin USA Inc., St. Peters, Missouri, USA) and tap water. Body weight ranged from 29 to $35 \mathrm{~kg}$ and remained constant throughout the study.

Surgical procedures. Chronic catheters $(0.13 \mathrm{~cm}$; Allegiance Healthcare, McGaw Park, Illinois, USA) were implanted at least 1 week prior to the first experiment. For blood sampling, an indwelling catheter was placed in the carotid artery. For the infusion of bupranolol, a second catheter was placed into the femoral vein and advanced into the inferior vena cava. All catheters were tunneled subcutaneously to the neck and exteriorized. Experimental protocol. Experiments were performed in the morning after a 24-hour fast. One hour prior to the beginning of blood sampling, an infusion $(30 \mathrm{ml} / \mathrm{h})$ of $0.9 \%$ saline $(n=7)$ or $1.5 \mu \mathrm{g} / \mathrm{kg} / \mathrm{min}$ bupranolol $(n=7$; kindly donated by Schwarz Pharma AG, Mannheim, Germany) was started.

To find the appropriate dosage of bupranolol, we tested two different doses (1.5 and $3.0 \mu \mathrm{g} / \mathrm{kg} / \mathrm{min}$ ) in a pilot study and determined the effect on plasma FFA. Augmentation of the initial dosage by $100 \%$ did not lead to a further decrease in FFA plasma concentration, indicating that $1.5 \mu \mathrm{g} / \mathrm{kg} / \mathrm{min}$ bupranolol was sufficient for maximum blockade of $\beta$-sympathetic effects on lipolysis.

For all experiments, arterial blood was sampled at 1-minute intervals for 120 minutes. Heart rate and blood pressure were monitored over the entire experimental period. Samples were collected in tubes containing EDTA and $0.275 \mathrm{mg} / \mathrm{ml}$ paraoxon, a lipoprotein lipase (LPL) inhibitor, to prevent in vitro lipolysis (18). Samples were immediately centrifuged, and the plasma was separated and stored at $-20^{\circ} \mathrm{C}$. The sampling period was reduced to 34 minutes during saline infusion in one experiment and to 54 and 72 minutes during infusion of bupranolol in two experiments due to catheter dysfunction.

Assays. FFAs (NEFA C; Wako Pure Chemical Industries, Richmond, Virginia, USA) and glycerol (GPOTrinder; Sigma Chemical Co., St. Louis, Missouri, USA) were measured by spectrophotometric analysis, as previously described (8). FFAs were measured in triplicate, with a coefficient of variation (CV) of $2.8 \%$, and glycerol was measured in duplicate $(\mathrm{CV}=5.4 \%)$.

Calculations. Time series analyses of FFA and glycerol concentration were performed using the software ULTRA (obtained from E. Van Cauter, University of Chicago Department of Medicine, Chicago, Illinois, USA) (19). To enhance sensitivity and specificity of our analysis, we applied a second mathematical approach for pulse detection and characterization: cluster analysis (sliding $t$ tests, obtained from M.L. Johnson, University of Virginia Department of Biomathematics, Charlottesville, Virginia, USA) (20). All analyses were performed on raw data. Cross-correlation analysis was used to determine the relationships between FFA and glycerol concentrations.

Deconvolution analysis. Plasma concentration of FFAs reflects a balance between two processes: release of FFAs into the vascular compartment and elimination from the vascular compartment by liver, skeletal muscle, and heart muscle (21). To quantify release of FFAs into the circulation we employed deconvolution software (DECONV_S, obtained from M.L. Johnson (22). Deconvolution analysis calculates the moment-by-moment rate of appearance of FFAs, which must have occurred to account for the kinetics of plasma FFA concentrations. To make this calculation it is necessary to have a priori knowledge of the kinetics of FFA disappearance from the plasma compartment. Plasma FFA kinetics have been repeatedly characterized in the canine model. FFA disappearance kinetics can be accurately represented by a single compartment $(23,24)$. Also, over a wide concentration range, FFA disappearance is linear in that a straight-line correlation exists between plasma FFA concentrations and the rate of FFA disappearance $(23,25)$. One study done in the 1960 s suggested that FFAs may leave and then reenter the plasma space and that a second decay process exists. However, the slower time constant is one to two orders of magnitude slower than the faster exponential process (26). It is unlikely that the much slower process would measurably alter the calculated rates of appearance in the present studies of relatively rapid oscillations with a cycle time of about 9 minutes. We have therefore represented FFA disappearance kinetics by a single exponential process.

Validation experiments. We examined the ability of deconvolution to accurately reconstruct the release rate of FFAs into plasma using three different approaches. 
FFA disappearance kinetics in the conscious dog model. To assess FFA half-time we injected $1.0 \mathrm{mmol}$ of octanoate during bupranolol infusion (to suppress endogenous FFA oscillations), and sampled blood at 1-minute intervals for 10 minutes $(n=2)$. The half-life of FFAs during bupranolol infusion was $2.77 \pm 0.01$ minutes and was equivalent to FFA half-life in previous studies. For example, Eaton et al. reported a half-life of $2.6 \pm 0.2$ minutes, and that reported by Riemens et al. in a more recent study was $2.8 \pm 0.7$ minutes $(26,27)$. In addition, the decay during the 10-minute experiments fit a single exponential with no significant residuals $(r=0.996)$.

Reconstruction. To validate the ability of the deconvolution method to detect and quantitatively estimate the oscillatory FFA release, four dogs were studied during infusion of known pulses of octanoic acid on top of a non-oscillatory baseline FFA level. Octanoic acid was infused in square-wave pulses of 30,90 , or 180 seconds delivered by a programmable Harvard pump with a nominal dose of $1.0 \mathrm{mmol}, 0.5 \mathrm{mmol}$, and $1.5 \mathrm{mmol}$ per pulse, respectively. Each pulse was followed by a 12minute period with one blood sample taken per minute from the carotid artery. FFA plasma concentrations and octanoate concentration in the infusates were measured and the recovery of known octanoic pulses with the deconvolution method was estimated.

The average volume of distribution for octanoate calculated from each individual experiment was $1.96 \pm 0.27$ liters. There was a strong agreement between the number of pulses $(4.25 \pm 0.6$ vs. $4.25 \pm 0.6, P=0.5)$, pulse interval $(12.9 \pm 0.04 \mathrm{~min}$ vs. $13.0 \pm 0 \mathrm{~min}, P=0.17)$, and pulse height $(0.86 \pm 0.20 \mathrm{mM}$ vs. $0.73 \pm 0.06 \mathrm{mM}$, $P=0.27$ ) between the deconvolved pulses of FFA release and the known infused octanoate pulses (Figure 1a). The integrated pulse mass of the deconvolved FFA pulses and the known amount of infused octanoic acid showed a strong correlation $(r=0.91$, Figure $1 \mathrm{~b})$, and the deconvolution program detected $83 \% \pm 6 \%$ of the infused pulses.

Impact of noise. To determine the stability of the deconvolution approach in the presence of random variation, we systematically added Gaussian noise to real non-oscillatory FFA time series we had obtained by the infusion of bupranolol ( $n=7$, see Results). The noise added was of the following magnitudes: \pm 0.05 , $\pm 0.10, \pm 0.15$, or $\pm 0.20 \mathrm{mM}$. Adding noise of the magnitude of \pm 0.05 and $\pm 0.10 \mathrm{mM}$, the deconvolution method detected only one false positive pulse per hour $(1.4 \pm 0.2$ and $1.1 \pm 0.8$, respectively). When noise of a higher magnitude $( \pm 0.15$ and $\pm 0.20 \mathrm{mM})$ was added to the data files, the program was not able to fit the data according to the kinetic model.

To quantify the impact of noise under our experimental conditions, we calculated the total variability $\left(\mathrm{CV}_{\text {total }}\right)$ present in the FFA concentrations of the non-oscillatory time series and then derived the components resulting from assay noise and non-assay uncertainties (biological noise, $\left.\mathrm{CV}_{\text {bio }}=\mathrm{CV}_{\text {total }}-\mathrm{CV}_{\text {assay }}\right)$. The biological noise in the non-oscillatory FFA time series was $4.9 \% \pm 0.7 \%$.
Statistics. Values are reported as mean \pm SEM. Comparisons of the saline and bupranolol infusions were made using the Student $t$ test $(P<0.05$ was considered statistically significant).

\section{Results}

Experiments were performed starting at 8 a.m. on dogs fasted for 24 hours. In the basal state during saline infusion, both FFA and glycerol exhibited distinct cycles (Figure 2a): FFA cycled with a frequency of seven pulses per hour, with pulse length of 8 minutes and pulse amplitude of $0.11 \mathrm{mM}$ (Table 1). Glycerol cycled with very similar temporal parameters: frequency of eight pulses per hour, and pulse length of 7 minutes. Glycerol amplitude was $0.05 \mathrm{mM}$ (Table 1). Results of time series analysis were similar with ULTRA or CLUSTER software program analysis. Cross correlation of the arterial FFA and glycerol data showed a strong correlation with a lag time not different from zero $(-0.29 \pm 1.43 \mathrm{~min}, P<0.05)$.

Deconvolution analysis revealed bursts of FFA release (Figure 3b, Table 2 ) with an average frequency of 9.5 pulses per 60 minutes, but with time-dependent variation in burst frequency that ranged from one to three cycles per 10 minutes (Figure 3c). The burst-like release of FFA was observed on top of a basal nonoscillatory release, with about $40 \%$ of FFA release occurring in pulses (Figure 4).

To test the hypothesis that oscillations of lipolysis are mediated by $\beta_{3}$-innervation of the adipose tissue, we intravenously infused the $\beta$-blocker bupranolol, which has a high affinity for $\beta_{3}$-receptors.

During saline and bupranolol infusion, the mean heart rate ( $92 \pm 2$ vs. $94 \pm 4$ beats per minute, respectively), systolic blood pressure (120 \pm 4 vs. $122 \pm 7 \mathrm{mmHg}$ ),

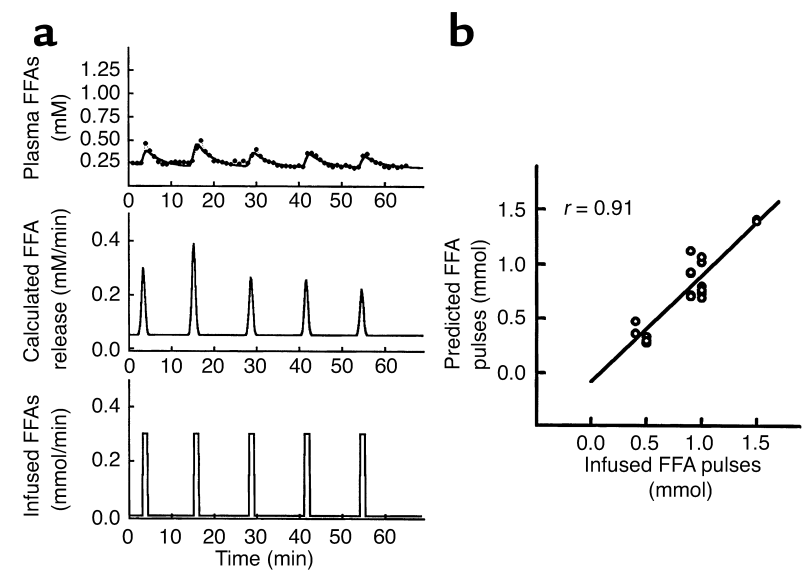

\section{Figure 1}

Reconstruction of infused FFA pulses with deconvolution analysis. (a) Upper graph shows the measured FFA concentration (filled circles) with best fit to concentration data (solid line). The profile of FFA release calculated by deconvolution analysis (middle graph) is in accordance with the infused FFA profile (five 90-second pulses with $0.5 \mathrm{mmol}$ octanoate per pulse, bottom graph). (b) A strong correlation exists between the predicted FFA pulses and actually infused octanoate pulses. 
(1)
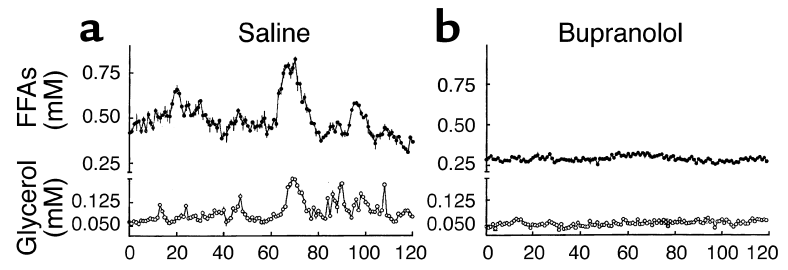

(2)
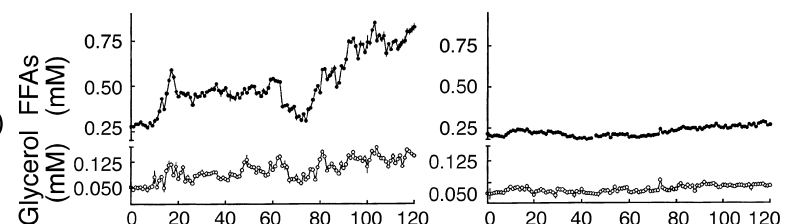

(3)
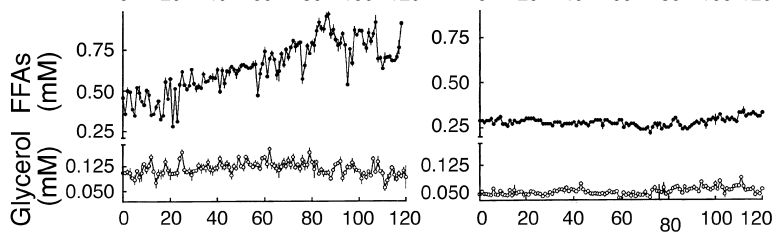

(4)

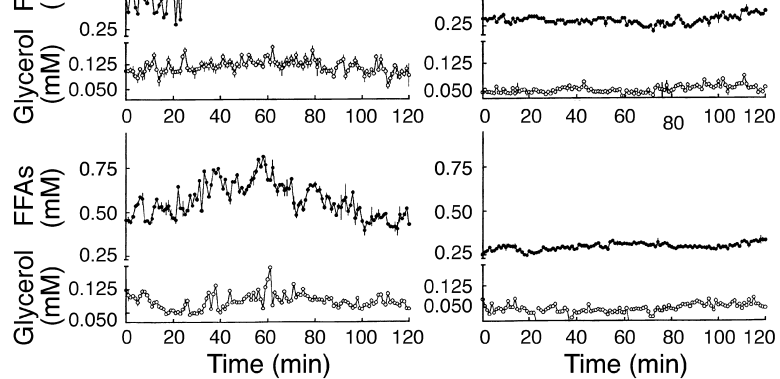

Figure 2

Time series of FFA (filled circles) and glycerol (open circles) plasma concentrations in four representative dogs. Oscillations of plasma FFAs and glycerol (a) were extinguished during bupranolol infusion (b). Data shown as mean \pm SEM.

and diastolic blood pressure $(89 \pm 4$ vs. $88 \pm 4 \mathrm{mmHg})$ were not significantly different between the groups (all $P \geq 0.05$ ). Additionally, there was no significant variation of blood pressure or heart rate within the individual experiments $(P \geq 0.05$ for all by ANOVA).

During bupranolol infusion, the plasma mean concentrations of FFA and glycerol were decreased by $50 \%$ ( $P<0.001$ and $P=0.02$, respectively) compared with saline controls (Table 1). Even though FFA and glycerol were not totally suppressed, the powerful, rapid oscillations in FFA and glycerol observed in the fasting state were extinguished with $\beta_{3}$-blockade of adipose tissue (Figure 2b). Pulse analysis of FFA and glycerol time series showed a decrease in pulse frequency $(P<0.001$ for both) and pulse amplitude $(P=0.005$ and $P=0.06$, respectively). In fact, very slow waves (two per hour for FFA and three per hour for glycerol) with a length of 35 minutes for FFA and 23 minutes for glycerol were detected (Table 1).

Deconvolution analysis revealed that during bupranolol infusion the burstlike release of FFAs was suppressed (Table 2). In contrast, the basal nonoscillatory release of FFAs was only marginally $(P=0.058)$ lower during bupranolol infusion $(0.064 \mathrm{mM} / \mathrm{min})$ compared with saline $(0.091 \mathrm{mM} / \mathrm{min})$.

\section{Table 1} concentration.
Although the pulsatile component of lipolysis vanished during bupranolol infusion $(P<0.001)$, the non-oscillatory release of FFAs (integrated over 120 minutes) was not significantly changed compared with the saline control experiments $(P=0.06$, Figure 4$)$.

\section{Discussion}

In the present study we show for the first time to our knowledge that FFA release in the fasting state consists of two clearly separable components: an oscillatory component that appears to be entirely dependent upon sympathetic innervation of adipose tissue, and a non-oscillatory, constitutive component that persists despite blockade of $\beta_{3}$-receptors. Results of our study derived from deconvolution analysis provide evidence that the non-oscillatory component of FFA release accounts for around $60 \%$ of the total lipid fuel release. On top of this stable component, approximately $40 \%$ of total FFA is released in bursts, with an average of nine pulses per hour. The frequency of appearance of these pulses is not constant, but varies as a function of time (from one to three pulses per 10 minutes), even under fasting conditions.

The observations of oscillatory FFA release and a pulsatile pattern of plasma FFAs and glycerol in the saline controls are in accordance with our previous study in which we showed rapid and significant oscillations in omental lipolysis (8). In the present case, rather than measuring FFA and glycerol release directly across the omental bed, we chose to determine the pattern of total body lipolysis by estimating the FFA release with deconvolution analysis. We were able to demonstrate that deconvolution can resolve exogenous pulses superseding a baseline in which oscillations were suppressed (see Methods). In addition, random "noise" added to $40 \%$ of the basal value was not detected as pulses. The results of validation experiments strongly support the use of deconvolution as an accurate approach to assessment of endogenous FFA release, similar to its exploitation for release of various hormones. (28-31). Yet it must be noted that FFA pulses in the validation experiments were simulated by infusion of octanoate, a medium-chain fatty acid that has the advantage of superior aqueous solubility but is certainly not representative of the physiological spectrum of FFAs in vivo, which are mainly

Plasma concentration and pulse analysis

\begin{tabular}{ccccc}
\hline & \multicolumn{2}{c}{ Saline $(n=7)$} & \multicolumn{2}{c}{ Bupranolol $(n=7)$} \\
& FFA & Glycerol & FFA & Glycerol \\
Plasma conc. $(\mathrm{mM})$ & $0.62 \pm 0.04$ & $0.12 \pm 0.02$ & $0.31 \pm 0.02^{\mathrm{B}}$ & $0.06 \pm 0.01^{\mathrm{A}}$ \\
Pulses (per 60 min) & $7.4 \pm 0.6$ & $8.4 \pm 0.7$ & $1.7 \pm 0.6^{\mathrm{B}}$ & $2.9 \pm 0.7^{\mathrm{B}}$ \\
Pulse length (min) & $8.2 \pm 0.8$ & $7.2 \pm 0.6$ & $35.3 \pm 5.6^{\mathrm{A}}$ & $23.5 \pm 4.9$ \\
Amplitude (mM) & $0.11 \pm 0.01$ & $0.05 \pm 0.01$ & $0.05 \pm 0.01^{\mathrm{A}}$ & $0.03 \pm 0.01^{\mathrm{A}}$
\end{tabular}

Data shown as mean \pm SEM. ${ }^{A}$ Value significantly different between saline and bupranolol $(P<0.05)$. BValue significantly different between saline and bupranolol $(P<0.001)$. Conc., 
(1)
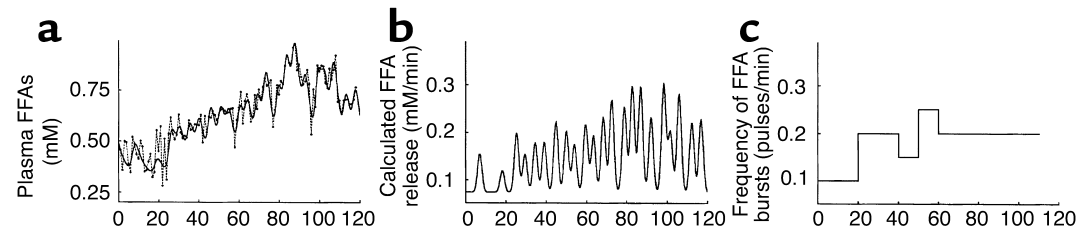

(2)
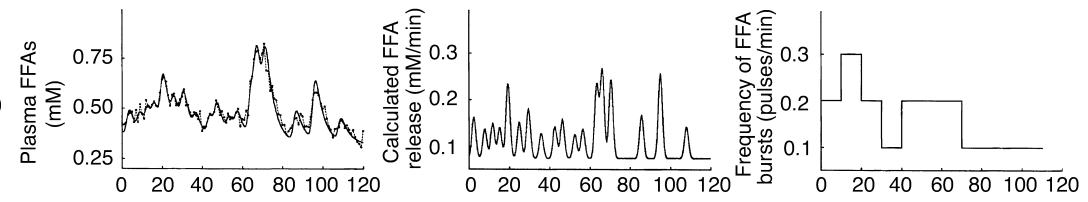

(3)
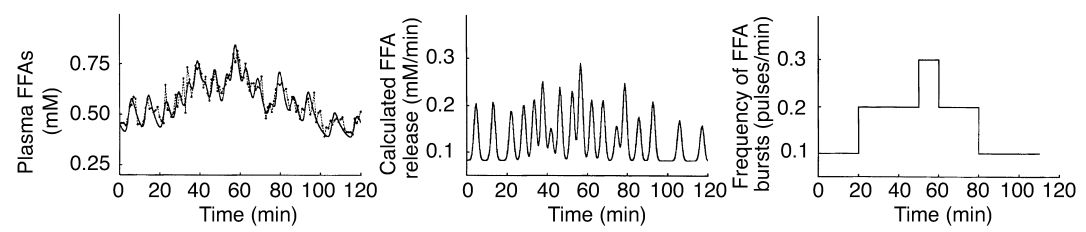

Figure 3

The profile of FFA release has been determined by deconvolution analysis. Data from three representative dogs are shown (numbered at left). (a) The experimentally observed data (filled circles) along with the calculated concentration profile corresponding to the best fit of the applied equations to the data (solid line). (b) Plots showing corresponding pattern ofFFA release, which exhibits rapid FFA bursts. (c) The highly variable burst frequency (calculated as pulses per minute at 10-minute intervals) as a function of time. long-chain fatty acids (32). Although our results suggest that the kinetic parameters of plasma octanoate were similar to those known for long-chain fatty acids, we did not directly compare pulsatile delivery of medium-chain fatty acids and long-chain fatty acids or validate the deconvolution approach against the isotope dilution method.

It is of interest to ask whether the oscillations of FFA appearance that we report in this study are generated by cyclic modulations of lipolysis rather than by variations in FFA uptake or reesterification in adipose tissue. That lipolysis is responsible is supported by others' kinetic data (tracer studies) as well as measures of arterio-venous difference across individual adipose beds, all of which indicate that the ratio of FFA to glycerol release is very close to the theoretical $3: 1$ during the postabsorptive period $(8,21,23,33)$. These earlier data indicate that there is little reesterification of FFAs or partial hydrolysis of triglycerides in the fasting state. Still, it is important to recognize that interpretation of the relationship between FFA and glycerol release measured in vivo is rather complex. The ratio may change under various conditions, including feeding, glucose infusion $(34,35)$, and exercise (23). Also when determined in regional tissue beds (35), the ratio between FFA appearance rate and glycerol appearance rate might differ from 3:1. Also, although we did not measure glycerol release directly in this study, we know from cross-correlation analysis of the glycerol and FFA plasma concentration time series that plasma FFA and glycerol clearly oscillate in phase. This observation does support cosecretion of FFAs and glycerol, as would be expected if oscillations were due to lipolysis (common to both metabolites). If clearance of either glycerol or FFAs (presumably unrelated mechanisms) were the cause for the observed oscillations, we would not expect exact co-oscillation. However, given limitations in interpretation of FFA/glycerol ratios, our data are consistent with assessment of lipolysis, but cannot be taken as conclusive proof.
It cannot be excluded here that the observed pulsatile pattern of plasma glycerol and FFAs is due to simultaneous cycles in FFA and glycerol clearance. However it is unlikely that oscillations of FFA plasma concentration are caused by variations in FFA uptake given the fact that the uptake of FFAs is strongly correlated to the FFA plasma concentration. Thus it can be assumed that under our experimental conditions the rate of FFA release reflects the rate of lipolysis and that the bursts of FFA release we report are due to bursts of lipolysis.

Oscillations of plasma FFAs and glycerol, as well as the deconvolved bursts of FFA release, were completely removed by $\beta_{3}$-blockade, indicating that the rapid bursts of lipolysis are generated by the sympathetic nervous system. A previous study failed to clarify the source of oscillatory lipolysis but ruled out that pulsatile secreted insulin is the driving force behind oscillation of lipolysis (8). When insulin oscillations were quenched with somatostatin and basal insulin was replaced, FFA and glycerol pulses were not reduced in fact there was some evidence for stronger cycles. The use of the generic $\beta$-blocker propranolol in that study yielded equivocal results (8). Although the regularity of the pulsatile profile was disrupted by propranolol, the average pulse frequency was not significantly different.

To directly examine the importance of the sympathetic nervous system in our present study we used bupranolol, a $\beta$-blocker with high affinity to $\beta_{3}$-receptors (36), to block the action of the sympathetic nervous system on adipose tissue lipolysis. $\beta_{3}$-receptors are

\section{Table 2}

FFA release calculated by deconvolution analysis

$\begin{array}{lcc} & \text { Saline }(n=7) & \text { Bupranolol }(n=7) \\ \text { Basal release }(\mathrm{mM} / \mathrm{min}) & 0.09 \pm 0.01 & 0.06 \pm 0.01^{\circ} \\ \text { Pulses (per 60 min) } & 9.5 \pm 0.8 & 1.8 \pm 0.3^{\mathrm{B}} \\ \text { Amplitude }(\mathrm{mM}) & 0.11 \pm 0.02 & 0.03 \pm 0.01^{\mathrm{A}}\end{array}$

Data shown as mean \pm SEM. AValue significantly different between saline and bupranolol $(P<0.05)$. ${ }^{B}$ Value significantly different between saline and bupra$\operatorname{nolol}(P<0.001)$. 


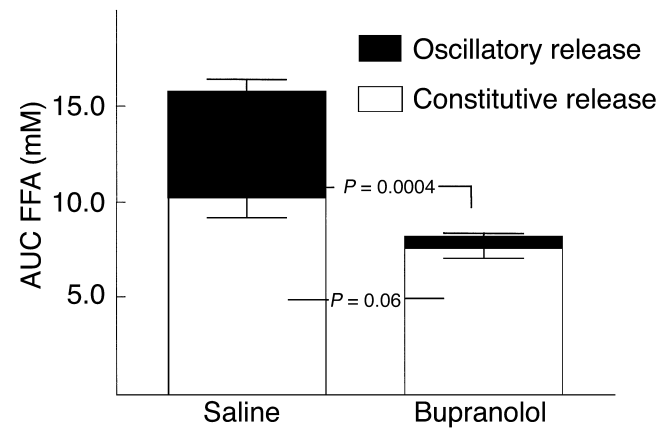

Figure 4

The pulsatile component of lipolysis is extinguished during $\beta$-blockade. The constitutive component of lipolysis does not significantly differ between saline and bupranolol experiments. Data show the calculated area under the curve $(A \cup C)$ of constitutive FFA release and FFA secretion pulses for 120 minutes during saline infusion $(n=7)$ and during bupranolol infusion $(n=7)$. Data shown as mean \pm SEM.

predominately expressed in fat cells. With $\beta_{3}$-receptors blocked, oscillations totally disappeared. It is to be expected that activation of lipolysis by catecholamines of neuronal origin is primarily mediated via $\beta_{3}$-receptors. First, these receptors are particularly responsive to high norepinephrine concentrations between sympathetic nerve varicosities and fat cell membranes at the synaptic cleft $(17,37)$. Second, these receptors are less implicated in desensitization (38). Besides $\beta$-adrenoceptors, dog adipose tissue also possesses $\alpha_{2}$-receptors, and $\beta$-blockade could theoretically lead to a spillover of catecholamines and activation of the antilipolytic $\alpha_{2}$ receptors. However, as opposed to human fat cells, in the dog, the number of $\alpha_{2}$ adrenergic binding sites is several times lower than that of $\beta$ sites (39). Furthermore, it has been shown in vivo that the $\alpha_{2}$ receptors in dog fat cells do not have physiological relevance for the modulation of lipolysis (39). Therefore it seems highly unlikely that $\alpha_{2}$ stimulation contributes to the observed suppression of lipolysis during $\beta$-blockade.

Blood flow has been demonstrated to be an important regulator of lipolysis (40), and it is possible that alteration in adipose tissue perfusion causes oscillations of FFA and glycerol plasma concentration and that the application of a $\beta$-blocker eliminates oscillatory or phasic activity of fat cell perfusion. It is indeed true that cyclic hemodynamic oscillations - under autonomic neural control - exist. However, even the less frequent fluctuations in regional perfusion, heart rate, or blood pressure interval (around $0.04-0.14 \mathrm{~Hz}$ ) can be differentiated from the observed oscillations of lipolysis (around $0.003 \mathrm{~Hz}$ ) (41). We did not measure blood flow in the present experiments. However, we have previously measured portal blood flow at 1minute intervals over 1 hour with an ultrasonic probe in conscious dogs $(n=4$, data not shown). We did not find evidence for oscillations in portal blood flow (as a reflection of pulsatility of the omental perfusion) at a frequency similar to that observed for FFA. Additionally, the results of the present study support a direct effect of the sympathetic nervous system on the fat cell rather than an indirect effect by changes in vascular perfusion of adipose tissue. First, we observed no changes in parameters of cardiovascular activity, heart rate, or blood pressure, indicating a low activity of bupranolol on cardiovascular $\beta$-receptors in the selected dose. Second, in our previous study, infusion of the $\beta_{1}$ - and $\beta_{2}$-receptor blocker propranolol in a threefold higher dose failed to completely suppress oscillatory lipolysis (8). If modulation of the vascular tone in the adipose tissue were the dominant mechanism for sympathetic alteration of lipolysis, we would expect stronger influence of the vasoactive $\beta$-blocker propranolol on lipolysis and oscillatory lipolysis in comparison with bupranolol. Compared with propranolol, bupranolol has a tenfold higher affinity for the $\beta_{3}$-receptor, which is not significantly expressed in the vascular system (42).

The results of this study show that under fasting conditions lipolysis is composed of two components: a basal constitutive, rather constant component independent of sympathetic outflow, plus a highly variable oscillatory component driven by the sympathetic nervous system. Constitutive lipolysis may be due to blood-borne lipolytic signals such as growth hormone, thyroid-stimulating hormone, or glucocorticoids acting to secure a minimum fuel supply for the organism under fasting conditions, when tissues are increasingly dependent on the breakdown of triglycerides because of fading glycogen stores $(43,44)$. Also, the non-oscillatory component could be due at least in small part to the breakdown of plasma triglycerides by lipoprotein lipase. Data about the contribution of LPL activity to plasma FFA concentrations in the fasting state are inconsistent $(21,45)$. A recent study suggests that LPL is more engaged in the storage of FFAs than in the release of lipid fuel into the circulation and that LPL activity leads more to an increase in glycerol, which can not be metabolized by adipose tissue, than to an increase in FFA (21). Thus, it appears unlikely that the constitutive component of lipolysis is due to LPL activity.

A recent study demonstrated oscillatory lipolysis in perifused isolated and denervated adipocytes (46). It is not clear whether there is a direct relationship between oscillatory behavior demonstrated in vitro and coordinated oscillations in the intact organism. Oscillatory lipolysis may be generated inside the adipocyte, and the CNS could synchronize the moment-to-moment alterations of lipolysis, generating powerful oscillations of FFA and glycerol in the systemic circulation. If that were true, then total suppression of the oscillations with the $\beta_{3}$-blocking agent could only be interpreted as a decoupling phenomenon; i.e., once the sympathetic nervous system is blocked, lipolysis from different adipose tissue beds could oscillate out of phase and cancel each other, so the ultimate outcome would appear to be non-oscillatory FFA concentrations. We previously 
showed lipolytic cycles in the visceral fat depot using arteriovenous difference measurements (8). That the visceral cycles appear to be in synchrony with other adipose depots suggests coordinated regulation. Of course it is also possible that the oscillatory component is contributed by one adipose tissue depot (visceral?), while the non-oscillatory component is contributed by another (subcutaneous?). Resolution of these questions will require invasive arteriovenous studies of different adipose tissue depots and further studies of in vitro oscillations with and without stimulation by sympathetic agonists.

Cyclicity is a characteristic shared by a variety of systems that are under control of the sympathetic nervous system, such as oscillations of skin sympathetic nerve activity (47) and sympathetic nerve discharge by central neurons (48). The activity of the sympathetic nervous system is directly influenced by the CNS (13), which plays an important role as the control center of energy homeostasis $(9,49)$. Here we show for the first time that oscillatory modulation of lipolysis may play an integral part in the control of moment-to-moment energy release from adipose stores and may supplement established functions of the CNS to control food intake and energy expenditure for the overall maintenance of energy balance. Beyond their functions in energy storage and fuel supply, FFAs have been shown to be a key regulator in a variety of metabolic processes (50). Hepatic glucose production seems to be under control of FFAs $(51,52)$, and a rise in FFA bursts could be at least a contributing factor to obesity-related insulin resistance. Insulin resistance is marked in upper body obesity but is mild or absent in lower body obesity (53), suggesting that increased delivery of FFAs from visceral adipose tissue to the liver may be largely responsible for the inappropriately elevated hepatic glucose output in the state of insulin resistance. It is of interest to clarify in further studies whether hepatic glucose output specifically depends on the pattern of burst-like regulation of visceral adipose tissue lipolysis and whether the burst-like and constitutive components of lipolysis are equally regulated at individual sites of adipose tissue. It will be essential to examine the regulation of moment-to-moment release of FFAs in individual fat depots.

In conclusion, in this study we show that release of energy from the adipose tissue is composed of a constitutive component independent of nervous stimulation, and an oscillatory component regulated by the sympathetic output of the CNS. Burst-like control of lipolysis may represent the efferent loop of the CNS to coordinate release of energy in appropriate accordance with energy demand and regulate the mass of stored body fat. It will be very important to identify the signals of the efferent loop to the CNS in this system and to study the impact of factors like leptin (54) and $\alpha$-melanocyte-stimulating hormone (55), which centrally raise sympathetic activity and might also modulate moment-to-moment release of energy from the adipose stores. Also, a preliminary report of oscillations in plasma FFA in humans has been published (56). Thus, parallel human studies will be of particular interest to understand the importance of FFA bursts in normal and pathological states including impaired glucose tolerance, type 2 diabetes, and obesity.

\section{Acknowledgments}

This work was supported by research grants from the NIH (DK-27619 and DK-29867). The authors extend extreme gratitude to E. Kirkman for her surgical expertise and to M. Ader, G. van Citters, and V. Ionut for their help and support.

1. Frayn, K.N. 1998. Regulation of fatty acid delivery in vivo. Adv. Exp. Med. Biol. 441:171-179.

2. Owen, O.E., Reichard, G.A., Jr., Patel, M.S., and Boden, G. 1979. Energy metabolism in feasting and fasting. Adv. Exp. Med. Biol. 111:169-188.

3. Coppack, S.W., Jensen, M.D., and Miles, J.M. 1994. In vivo regulation of lipolysis in humans. J. Lipid Res. 35:177-193.

4. Kuzawa, C.W. 1998. Adipose tissue in human infancy and childhood: an evolutionary perspective. Am. J. Phys. Anthropol. 27(Suppl.):177-209.

5. Boden, G. 2001. Free fatty acids-the link between obesity and insulin resistance. Endocr. Pract. 7:44-51

6. Frayn, K.N., Williams, C.M., and Arner, P. 1996. Are increased plasma non-esterified fatty acid concentrations a risk marker for coronary heart disease and other chronic diseases? Clin. Sci. (Lond.) 90:243-253.

7. Arner, P. 1996. Regulation of lipolysis in fat cells. Diabetes Reviews. 4:450-463.

8. Getty, L., Panteleon, A.E., Mittelman, S.D., Dea, M.K., and Bergman, R.N. 2000. Rapid oscillations in omental lipolysis are independent of changing insulin levels in vivo. J. Clin. Invest. 106:421-430.

9. Schwartz, M.W., Woods, S.C., Porte, D., Jr., Seeley, R.J., and Baskin, D.G. 2000. Central nervous system control of food intake. Nature. 104:661-671.

10. Kim, M.S., et al. 2000. The central melanocortin system affects the hypothalamo-pituitary thyroid axis and may mediate the effect of leptin. J. Clin. Invest. 105:1005-1011.

11. Bartness, T.J., and Bamshad, M. 1998. Innervation of mammalian white adipose tissue: implications for the regulation of total body fat. Am. J. Physiol. 275:R1399-R1411.

12. Nonogaki, K. 2000. New insights into sympathetic regulation of glucose and fat metabolism. Diabetologia. 43:533-549.

13. Bamshad, M., Aoki, V.T., Adkison, G., Warren, W.S., and Bartness, T.J. 1998. Central nervous system origins of the sympathetic nervous system outflow to white adipose tissue. Am. J. Physiol. 275:R291-R299.

14. Shi, H., and Bartness, T.J. 2001. Neurochemical phenotype of sympathetic nervous system outflow from brain to white fat. Brain Res. Bull. 54:375-385.

15. Paschoalini, M.A., and Migliorini, R.H. 1990. Participation of the CNS in the control of FFA mobilization during fasting in rabbits. Physiol. Behav. 47:461-465.

16. Barkai, A., and Allweis, C. 1972. Effect of electrical stimulation of the hypothalamus on plasma free fatty acid concentration in cats. J. Lipid Res. 13:725-732.

17. Strosberg, A.D. 1997. Structure and function of the beta 3-adrenergic receptor. Annu. Rev. Pharmacol. Toxicol. 37:421-450.

18. Zambon, A., Hashimoto, S.I., and Brunzell, J.D. 1993. Analysis of techniques to obtain plasma measurement of levels of free fatty acids. J. Lipid Res. 34:1021-1028.

19. van Cauter, E. 1988. Estimating false-positive and false-negative errors in analyses of hormonal pulsatility. Am. J. Physiol. 254:E786-E794.

20. Johnson, M.L., and Veldhuis, J.D. 1986. Cluster analysis: a simple, versatile and robust algorithm for endocrine pulse detection. Am. J. Physiol. 250:E486-E493.

21. Coppack, S.W., Persson, M., Judd, R.L., and Miles, J.M. 1999. Glycerol and nonesterified fatty acid metabolism in human muscle and adipose tissue in vivo. Am. J. Physiol. 276:E233-E240.

22. Johnson, M.L., and Veldhuis, J.D. 1995. Evolution of deconvolution analysis as a hormone pulse detection method. Methods in Neuroscience. 28:1-24.

23. Shaw, W.A., Issekutz, T.B., and Issekutz, B., Jr. 1975. Interrelationship of FFA and glycerol turnovers in resting and exercising dogs. J. Appl. Physiol. 39:30-36.

24. Miles, J.M., Ellman, M.G., McClean, K.L., and Jensen, M.D. 1987. Validation of a new method for determination of free fatty acid turnover. Am. J. Physiol. 252:E431-E438. 
25. Armstrong, D.T., et al. 1961. Regulation of plasma free fatty acid turnover. Am. J. Physiol. 201:9-15.

26. Eaton, R.P., Berman, M., and Steinberg, D. 1969. Kinetic studies of plasma free fatty acid and triglyceride metabolism in man. J. Clin. Invest. 48:1560-1579.

27. Riemens, S.C., et al. 1998. Measurement of free fatty acid kinetics during non-equilibrium tracer conditions in man: implications for the estimation of the rate of appearance of free fatty acids. Eur. J. Clin. Invest. 28:108-114

28. Veldhuis, J.D., Carlson, M.L., and Johnson, M.L. 1987. The pituitary gland secretes in bursts: appraising the nature of glandular secretory impulses by simultaneous multiple-parameter deconvolution of plasma hormone concentrations. Proc. Natl. Acad. Sci. USA. 84:7686-7690.

29. Gasperi, M., et al. 2002. GH secretion is impaired in patients with primary hyperparathyroidism. J. Clin. Endocrinol. Metab. 87:1961-1964.

30. Juhl, C., et al. 2002. Effects of fasting on physiologically pulsatile insulin release in healthy humans. Diabetes. 51(Suppl. 1):S255-S257.

31. Van Dam, E.W., et al. 2002. Increase in daily LH secretion in response to short-term calorie restriction in obese women with PCOS. Am. J. Physiol. Endocrinol. Metab. 282:E865-E872.

32. Miller, H.I., Gold, M., and Spitzer, J.J. 1962. Removal and mobilization of individual FFA in dogs. Am. J. Physiol. 202:370-374.

33. Wolfe, R.R., and Peters, E.J. 1987. Lipolytic response to glucose infusion in human subjects. Am. J. Physiol. 252:E218-E223.

34. Leibel, R.L., Hirsch, J., Berry, E.M., and Gruen, R.K. 1985. Alterations in adipocyte free fatty acid re-esterification associated with obesity and weight reduction in man. Am. J. Clin. Nutr. 42:198-206.

35. Jensen, M.D. 1999. Regional glycerol and free fatty acid metabolism before and after meal ingestion. Am. J. Physiol. 276:E863-E869.

36. Galitzky, J., et al. 1993. Coexistence of beta $1^{-}$, beta $2^{-}$, and beta $3_{3}$-adrenoceptors in dog fat cells and their differential activation by catecholamines. Am. J. Physiol. 264:E403-E412.

37. Collins, S., and Surwit, R.S. 2001. The beta-adrenergic receptors and the control of adipose tissue metabolism and thermogenesis. Recent Prog. Horm. Res. 56:309-328.

38. Carpene, C., et al. 1993. Desensitization of beta-1 and beta-2, but not beta-3, adrenoceptor-mediated lipolytic responses of adipocytes after long-term norepinephrine infusion. J. Pharmacol. Exp. Ther. 265:237-247.

39. Taouis, M., Berlan, M., Montastruc, P., and Lafontan, M. 1988. Mechanism of the lipid-mobilizing effect of alpha-2 adrenergic antagonists in the dog. J. Pharmacol. Exp. Ther. 247:1172-1180.

40. Edens, N.K., Leibel, R.L., and Hirsch, J. 1990. Mechanism of free fatty acid re-esterification in human adipocytes in vitro. J. Lipid Res. 31:1423-1431
41. van de Borne, B.P., Montano, N., Pagani, M., Oren, R., and Somers, V.K 1997. Absence of low-frequency variability of sympathetic nerve activity in severe heart failure. Circulation. 95:1449-1454.

42. Atgie, C., D’Allaire, F., and Bukowiecki, L.J. 1997. Role of beta1- and beta3-adrenoceptors in the regulation of lipolysis and thermogenesis in rat brown adipocytes. Am. J. Physiol. 273:C1136-C1142.

43. Boyle, P.J., et al. 1992. Role of GH in regulating nocturnal rates of lipolysis and plasma mevalonate levels in normal and diabetic humans. Am. J. Physiol. 263:E168-E172.

44. Pucci, E., Chiovato, L., and Pinchera, A. 2000. Thyroid and lipid metabolism. Int. J. Obes. Relat. Metab. Disord. 24(Suppl. 2):S109-S112.

45. Samra, J.S., Clark, M.L., Humphreys, S.M., Macdonald, I.A., and Frayn, K.N. 1996. Regulation of lipid metabolism in adipose tissue during early starvation. Am. J. Physiol. 271:E541-E546.

46. Getty, L., Richard, A.M., and Corkey, B.E. 2000. Rapid oscillatory lipolysis in isolated perifused adipocytes. Obes. Res. 8(Suppl. 1):22S. (Abstr.)

47. Cogliati, C., Magatelli, R., Montano, N., Narkiewicz, K., and Somers, V.K. 2000. Detection of low- and high-frequency rhythms in the variability of skin sympathetic nerve activity. Am. J. Physiol. Heart Circ. Physiol. 278:H1256-H1260.

48. Ando, S., et al. 1994. Postganglionic sympathetic nerve discharges can contain both central and pulse-related oscillations simultaneously in rabbits. J. Auton. Nerv. Syst. 47:189-199.

49. Kaiyala, K.J., Woods, S.C., and Schwartz, M.W. 1995. New model for the regulation of energy balance and adiposity by the central nervous system. Am. J. Clin. Nutr. 62:1123S-1134S.

50. Bergman, R.N., et al. 2001. Central role of the adipocyte in the metabolic syndrome. J. Investig. Med. 49:119-126.

51. Rebrin, K., Steil, G.M., Getty, L., and Bergman, R.N. 1995. Free fatty acid as a link in the regulation of hepatic glucose output by peripheral insulin. Diabetes. 44:1038-1045.

52. Rebrin, K., Steil, G.M., Mittelman, S.D., and Bergman, R.N. 1996. Causal linkage between insulin suppression of lipolysis and suppression of liver glucose output in dogs. J. Clin. Invest. 98:741-749.

53. Bjorntorp, P. 1991. Metabolic implications of body fat distribution. Diabetes Care. 14:1132-1143.

54. Haynes, W.G., Morgan, D.A., Walsh, S.A., Mark, A.L., and Sivitz, W.I. 1997. Receptor-mediated regional sympathetic nerve activation by leptin. J. Clin. Invest. 100:270-278.

55. Dunbar, J.C., and Lu, H. 2000. Proopiomelanocortin (POMC) products in the central regulation of sympathetic and cardiovascular dynamics: studies on melanocortin and opioid interactions. Peptides. 21:211-217.

56. Eisner, J.R., Huecking, K., Bergman, R.N., and Dunaif, A. 2001. Free fatty acid oscillations in humans: correlation with adiposity. 83rd Annual Meeting of The Endocrine Society. Denver, Colorado, USA. 3-67. 